\title{
Effect of Dolichos biflorus Seeds Based Functional Beverage on In Vitro Calcium Oxalate Crystallization in Human Urine
}

\author{
Ravi Kant ${ }^{1}$, Thakur Gurjeet Singh ${ }^{1, * \text { (D) }}$ \\ 1 Chitkara College of Pharmacy, Chitkara University, Punjab, 140401, India; \\ * Correspondence: gurjeet.singh@ @ chitkara.edu.in (T.G.S.);
}

Scopus Author ID 27667828700

Received: 20.08.2021; Revised: 25.09.2021; Accepted: 29.09.2021; Published: 24.10.2021

\begin{abstract}
Calcium oxalate is the most common type of urolithiasis. The crystallization process includes nucleation, growth, and the aggregation of crystals. This study has used Dolichos biflorus seeds as a functional beverage to explore the role of its bioactive substances on the crystallization process of calcium oxalate in managing urolithiasis. A human urine model of in vitro calcium oxalate crystals was used in the study. Phytochemical screening of Functional beverage of Dolichos biflorus seeds was performed, and antioxidant activity was evaluated by measuring DPPH radical-scavenging activity, reducing power assay, and Hydrogen peroxide scavenging activity. Functional beverage of Dolichos biflorus seeds inhibited crystallization process by reducing aggregation of calcium oxalate crystals. The reduction in crystals aggregation helps prevent urolithiasis by keeping the crystals dispersed in the urine, controlling their size, and facilitating expulsion from the urinary tract. The results showed that the Functional beverage of Dolichos biflorus seeds has a significant quantity of flavonoids, glycosides, etc., and also possesses a significant antioxidant activity as evaluated by employing different antioxidant assays. Therefore, our findings suggested that the functional beverage of Dolichos biflorus seeds exhibited antiurolithiatic activity through inhibition of the crystallization process of the calcium oxalate process and significant antioxidant potential.
\end{abstract}

Keywords: Dolichos biflorus; calcium oxalate; urolithiasis; antiurolithiatic; functional beverage; crystallization; antioxidant activity.

(C) 2021 by the authors. This article is an open-access article distributed under the terms and conditions of the Creative Commons Attribution (CC BY) license (https://creativecommons.org/licenses/by/4.0/).

\section{Introduction}

Urolithiasis, the process of stone formation in the urinary tract, has tormented humans from ancient times. Paleopathology reported the incidence of urolithiasis from 7000 years back in preserved mummies throughout the world [1]. Urolithiasis may occur at any age, although onset is observed more in young and middle-aged adults [2]. In industrialized countries, 10$12 \%$ of predominantly male people have urolithiasis during their lives $[3,4]$. Treatment for renal stones recurrence may eventually lead to deterioration of renal function [5]. Among various types of urolithiasis, calcium oxalate $(75 \%$ - 90\%) is the most common type of stone afflicts the Asian population. Its proportion has increased in countries like China, Japan, India, and Indonesia [6].

Pharmacological and surgical therapeutic procedures have limitations as these do not overcome the risk of stone recurrence, and treatment like ESWL may also cause hypertension, renal damage, and impaired functioning [7]. A few years back, medicinal plants were proposed 
for better management of urolithiasis [8]. Till time plenty of research has been done in this discipline but could not provide satisfactory results. Altogether, few adverse events are reported using compounds extracted from medicinal plants $[9,10]$. With the recent developments in nutrition science, it was suggested as "Let functional food be used as medicine" [11], and thus, there is a requirement to switch from the nutritional aspect of food to functional foods as form of dietary therapy to cure diseases [12]. In our Indian traditional medicine, seeds of Dolichos biflorus (used as food pulse known as kultha/kulthi/horse gram) are traditionally used to treat urolithiasis $[13,14]$ with urinary diseases. Dolichos biflorus seeds are rich sources of various natural bioactive substances and can treat various diseases [15]. Many authors reported the in vitro effect of Dolichos biflorus seeds on the crystallization of calcium phosphate [16- 20]. However, the specific mechanism of Dolichos biflorus seeds and its effect in human urine calcium oxalate crystal model is yet to be explored; therefore, we planned this study and used Dolichos biflorus seeds as a functional beverage to explore the role of bioactive substances of Dolichos biflorus seeds on the crystallization of calcium oxalate urolithiasis.

The formation of urinary calcium oxalate crystals involves a crystallization process that includes nucleation, growth, and the aggregation of crystals [21, 22]. Therefore, to explore the mechanism of Dolichos biflorus we evaluated its effect on in vitro crystallization of calcium oxalate urolithiasis in human urine. Oxidative stress occurs due to the presence of oxidants exceeds the concentration of antioxidants, resulting in the production of free radicals. In vitro, animal, and human research all support the active role of oxidative stress in the production of urinary stones [3]. Lithogenic crystals generated in urine directly stimulate the formation of reactive oxygen species (ROS) in renal tubular cells, resulting in oxidative damage and the release of pro-inflammatory mediators [4]. The formulation is used as a functional beverage in tea bags, as tea is a widely consumed worldwide [23]. We added some other dietary compounds to make them more palatable. So the antioxidant and anti-inflammatory potential of Functional Beverage of Dolichos biflorus and its constituents can be used as a novel herbal formulation to treat urolithiasis.

\section{Materials and Methods}

\subsection{General experimental procedure.}

The chemicals and solutions were of analytical grade, and all the reagents were freshly prepared before use. All constituents of study formulations were collected from Dehradun, Uttarakhand, during June 2017. The voucher specimen of all constituents was fixed into herbarium sheet and deposited at the College of Pharmacy, Chitkara University, Rajpura, for further reference. All botanical parts of the study formulation were powdered, passed through a mesh 60, and stored in an airtight container before extraction.

\subsection{Preparation of functional beverage (FB).}

Study formulation was assessed at various concentrations that is $0.5,1.0,1.5,2.0,2.5$ and $3.0 \mathrm{~g}$ per $250 \mathrm{~mL}$. Each tea bag was constituted of: moderately coarse powder of Dolichos biflorus (seeds) $(60 \% \mathrm{w} / \mathrm{w})$, Phyllanthus emblica L. (dried fruit) $(10 \% \mathrm{w} / \mathrm{w})$, Ocimum tenuiflorum L. (dried leaves) (10\% w/w), Green Tea (dried leaves) (8\% w/w), Withania somnifera (dried root powder) (5\% w/w), Foeniculum vulgare Mill. (Seed's powder) (5\% 
$\mathrm{w} / \mathrm{w})$ and Stevia (dried leaves) $(2 \% \mathrm{w} / \mathrm{w})$. The filled tea bag was dipped in $250 \mathrm{ml}$ hot water (temperature $90^{\circ} \mathrm{C}$ ) for 10 minutes. Teabag was removed after 10 minutes, and the extract was allowed to cool (at room temperature), filtered with filter paper, and used for antiurolithiatic activity.

\subsection{Calcium oxalate crystallization.}

Antiurolithiasis activity of Dolichos biflorus based functional beverage was evaluated on calcium oxalate crystals induced in the normal human's urine. The study protocol and all the experimental procedures used in this study were approved by the Institutional Ethics Committee (ISFCP/IEC/2019/41).

Calcium oxalate precipitation was induced by adding $40 \mu \mathrm{L}$ of $0.1 \mathrm{M}$ sodium oxalate per $\mathrm{ml}$ of urine (corresponding to $0.536 \mathrm{mg}$ ), every 30 minutes, for a total duration of the experiment 90 minutes at the four-time point, under shaking incubator at $37^{\circ} \mathrm{C}$, resulting in a final concentration of urine $(2-2.5 \mathrm{ml})$. Two aliquots of urine sample were used for the study, one as the control sample without formulation and the second with Dolichos biflorus based functional beverage for checking induction of calcium oxalate precipitation to check the crystallization process. Sodium oxalate was added $30 \mathrm{~min}$ before the crystallization process. Antiurolithiasis activity of Dolichos biflorus based functional beverage was assessed at various concentrations of $0.5,1.0,1.5,2.0,2.5$, and $3.0 \mathrm{~g}$ per $250 \mathrm{~mL}$, based on a dose-response curve.

\subsection{Collection and analysis of urine.}

Urine samples were obtained from 10 healthy subjects ( 05 males and 05 females) with no urolithiasis personal or family history. 24-hour urine samples were collected from each subject in provided sterile polypropylene urine bottles. The calcium oxalate crystals obtained were analyzed at 0 and $24 \mathrm{~h}$ after the crystallization process. The turbidimetric assay was used for the semiquantitative analysis of crystals [24]. For the post crystallization process, urine sample treated with Dolichos biflorus based functional beverage and without treatment sample centrifuged for five minutes at $3000 \mathrm{rpm}$. The urine supernatant was placed on the Neubauer chamber, and a qualitative analysis of crystals was performed. Crystal images were recorded with a digital video camera associated with a light microscope (Olympus upright microscopes, USA).

\subsection{Assessment of in vitro antioxidant assay.}

Standard methods for reducing power, DPPH (2,2-diphenyl-1-picrylhydrazyl) radical scavenging, and hydrogen peroxide radical scavenging were used for evaluating antioxidant potential of Dolichos biflorus based functional beverage comparison with ascorbic acid $[25,26]$.

\subsection{Phytochemical analysis.}

The phytochemical analysis of Dolichos biflorus based functional beverage was determined as per the standard methods described in Ayurvedic Pharmacopoeia of India to determine chemical constituents present in it, like saponins phenolic compounds, glycosides, flavones and etc. (Table 1). 


\subsection{Statistical analysis.}

Values were expressed in terms of mean \pm standard error mean. Differences among data were determined using a one-way ANOVA test followed by Tukey's multiple comparison test (GraphPad Software, Inc., version 6, CA, USA.), and $\mathrm{P}<0.05$ was considered statistically significant.

\section{Results and Discussion}

\subsection{Effect on calcium oxalate crystallization.}

Based on the effect of various dosages of the study formulation on the turbidity of the solution, a dose-response curve was built using human urine. When illustrated in Figure 1, the absorbance of the solution reduced as the study formulation dose was increased. Doses above $2 \mathrm{~g} / 250 \mathrm{~mL}$ were not able to induce a further change in absorbance, and therefore this dose was used in the subsequent experiments. The decrease in absorbance indicates a lower density of crystals. However, the observation of crystals by light microscopy showed that increasing doses of study formulation produced a lower crystal density and smaller crystals (Figure $2 \&$ 3). After 24-h of crystallization, an intense aggregation of calcium oxalate crystals was observed in control urine (Figure 2), whereas this phenomenon was considerably inhibited in the presence of study formulation (Figure 3).

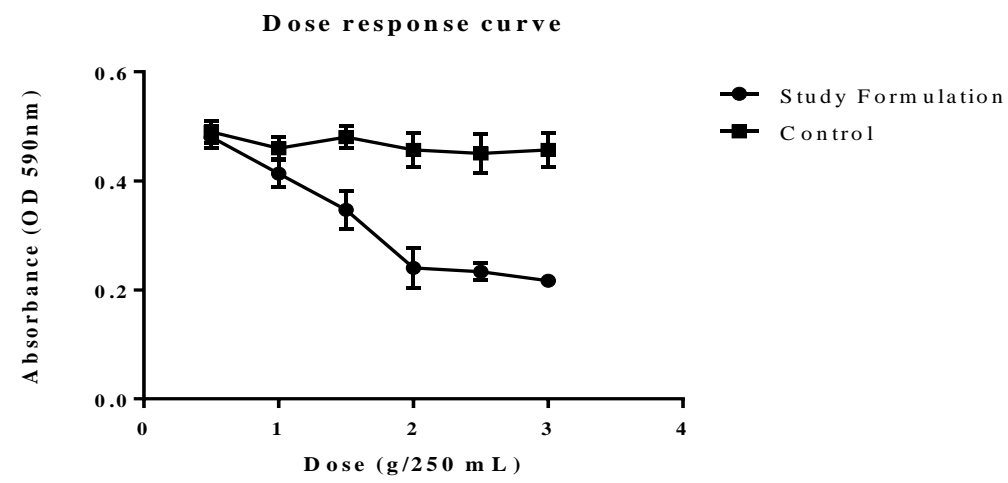

Figure 1. Dose-response curve for the effect of different doses of study formulation on the turbidity of the solution of Dolichos biflorus based functional beverage.

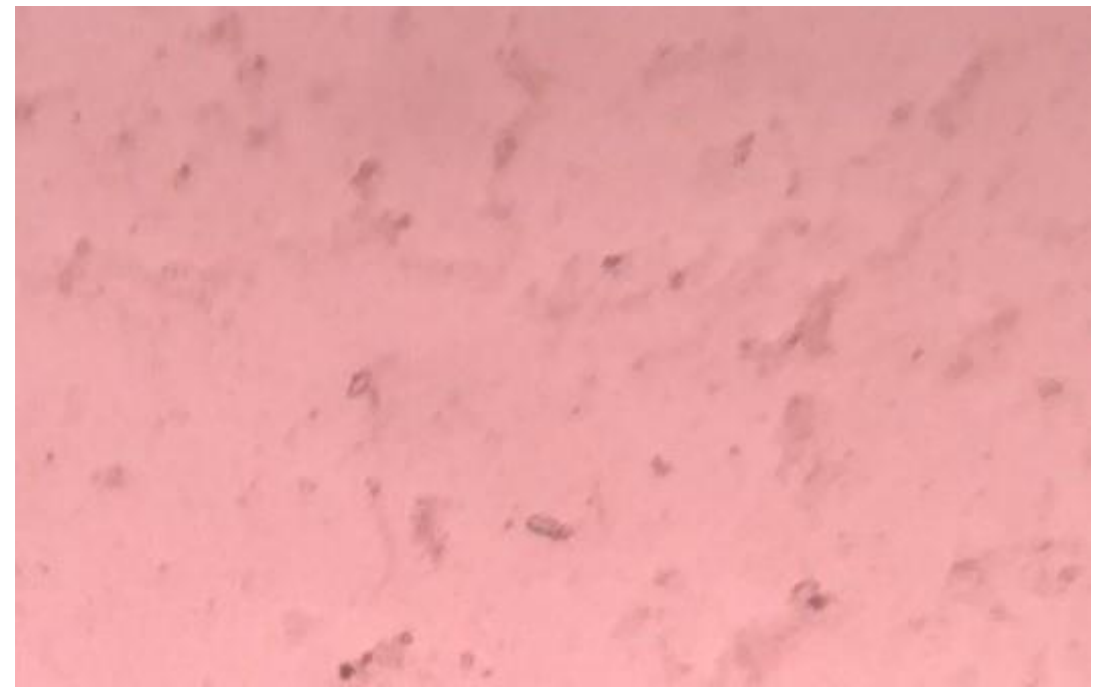

Figure 2. Intense aggregation of calcium oxalate crystals in control urine. 


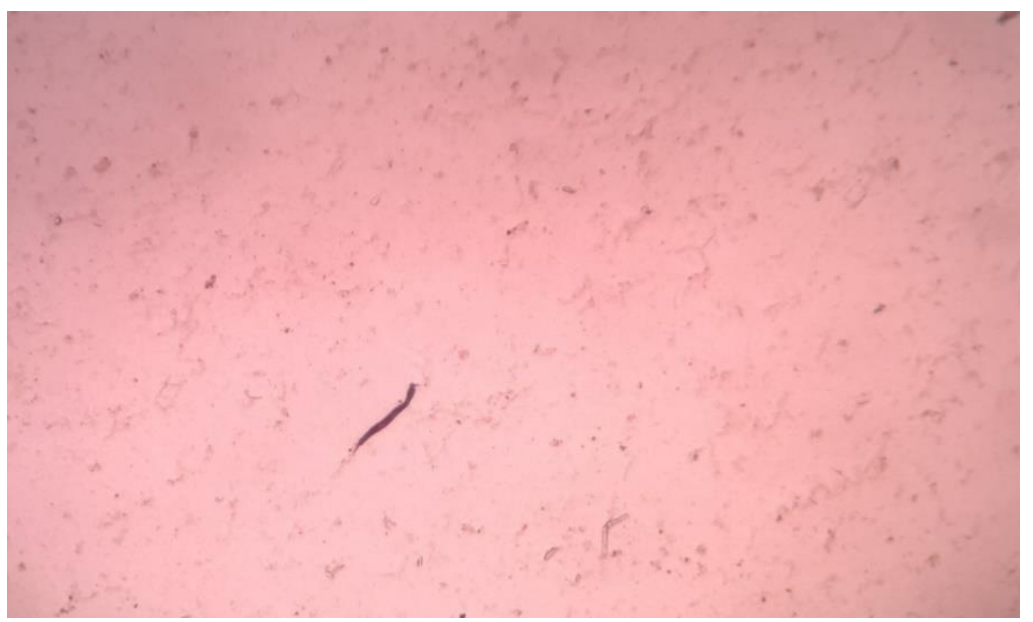

Figure 3. Inhibition of the aggregation of calcium oxalate crystals in study formulation treated urine.

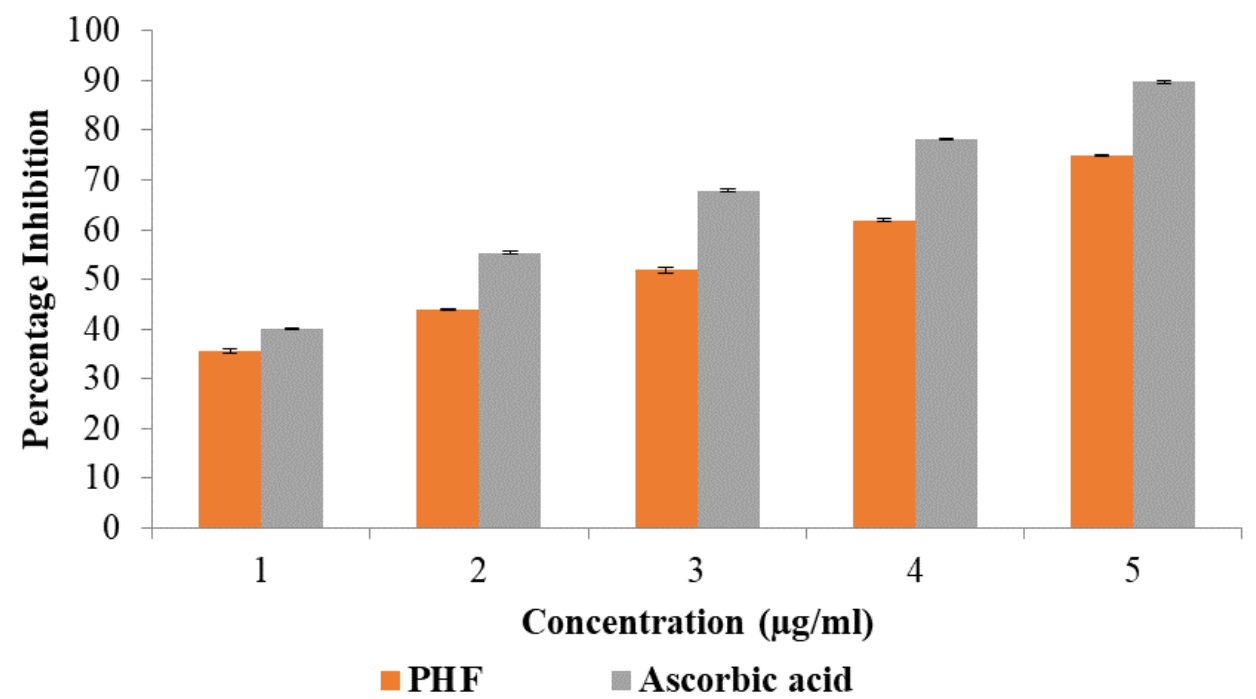

Figure 4. Assessment of DPPH scavenging activity of Dolichos biflorus based functional beverage.

\subsection{Assessment of antioxidant activity.}

\subsubsection{DPPH assay.}

Antioxidant activity of polyherbal formulation was evaluated by employing DPPH scavenging assay. Different concentrations of polyherbal formulation $(1-5 \mu \mathrm{g} / \mathrm{ml})$ were used to determine the DPPH scavenging activity. Dose-dependent percent inhibition was observed with different concentrations of the polyherbal formulation. At the concentration of $1 \mu \mathrm{g} / \mathrm{ml}$ percent inhibition was found to be 35.56 whereas, in the case of $2 \mu \mathrm{g} / \mathrm{ml}, 3 \mu \mathrm{g} / \mathrm{ml}, 4 \mu \mathrm{g} / \mathrm{ml}$, and $5 \mu \mathrm{g} / \mathrm{ml}$ percent inhibition was found to be to $43.97,43.92,51.90 .61 .94$ and 74.92 respectively. The IC50 of the polyherbal formulation was found to be 2.622. In the case of ascorbic acid (used as standard compound), percent inhibition at different concentrations $1 \mu \mathrm{g} / \mathrm{ml}-5 \mu \mathrm{g} / \mathrm{ml}$ was found to be 41.12, 55.32, 67.86. 78.23 and 89.69, respectively, and IC50 value was found to be 1.669 (Figure 4).

\subsubsection{Reducing power assay.}

This assay was done with different concentration viz $12.5 \mu \mathrm{g} / \mathrm{ml}, 25 \mu \mathrm{g} / \mathrm{ml}, 50 \mu \mathrm{g} / \mathrm{ml}$, $100 \mu \mathrm{g} / \mathrm{ml}, 200 \mu \mathrm{g} / \mathrm{ml}, 400 \mu \mathrm{g} / \mathrm{ml}, 800 \mu \mathrm{g} / \mathrm{ml}$ for both Polyherbal formulation and standard ascorbic acid. Polyherbal shows the absorbance of 0.266 for concentration $12.5 \mu \mathrm{g} / \mathrm{ml}$ and 
2.081 for concentration $800 \mu \mathrm{g} / \mathrm{ml}$ whereas for ascorbic acid absorbance at $12.5 \mu \mathrm{g} / \mathrm{ml}$ was found to be 0.306 and 2.422 for concentration $800 \mu \mathrm{g} / \mathrm{ml}$. EC50 was calculated and $61.81 \mu \mathrm{g} / \mathrm{ml}$ for Polyherbal formulation and $38.42 \mu \mathrm{g} / \mathrm{ml}$ for ascorbic acid (Figure 5).

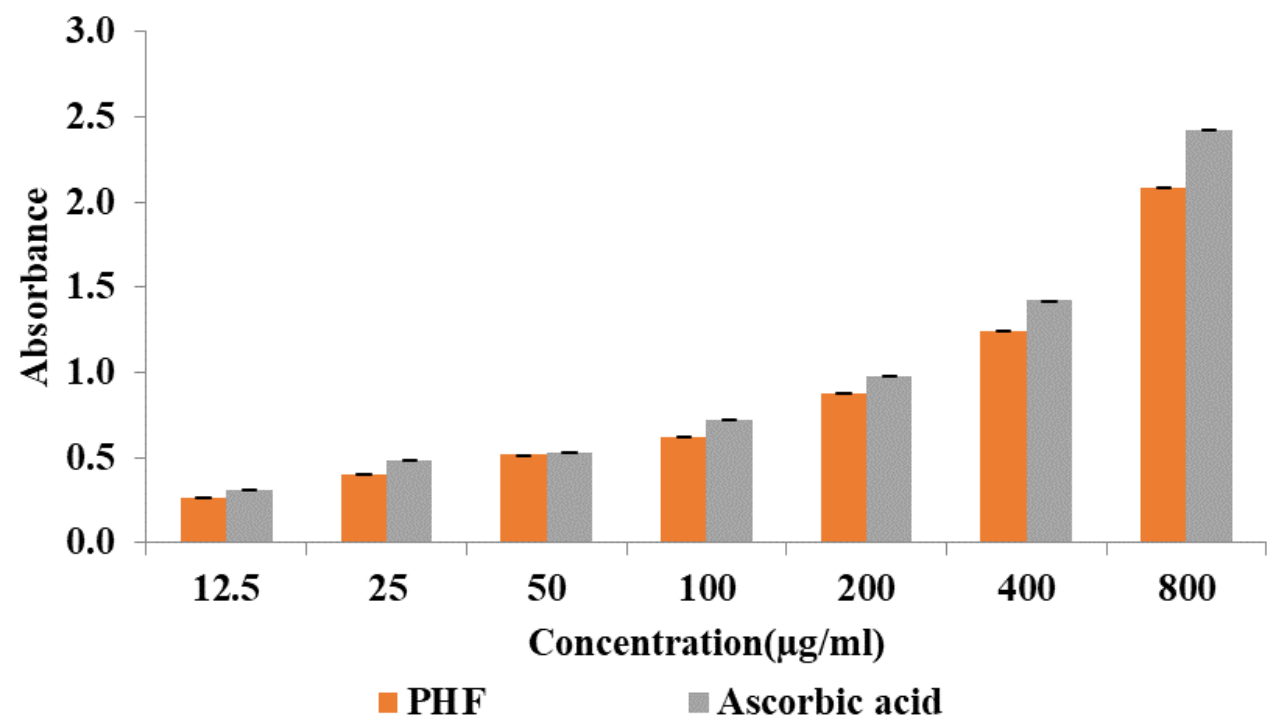

Figure 5. Assessment of Reducing power activity of Dolichos biflorus based functional beverage.

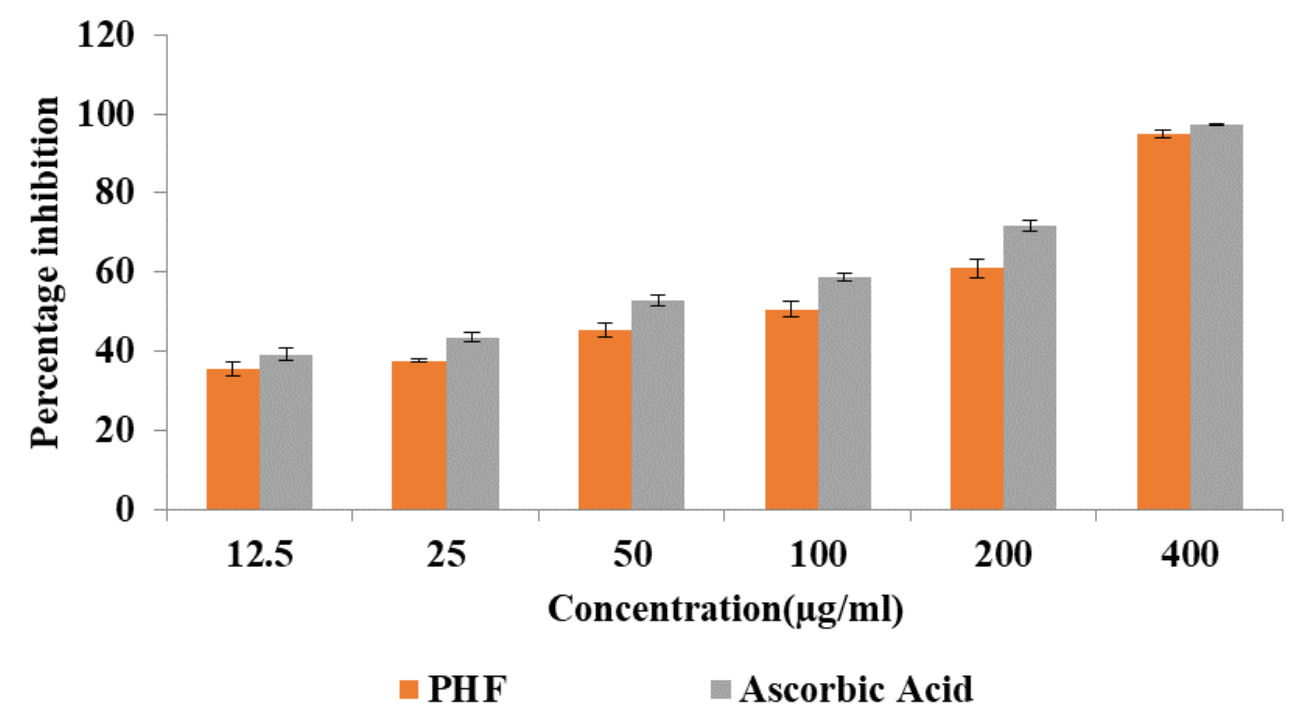

Figure 6. Assessment of Hydrogen peroxide scavenging activity of Dolichos biflorus based functional beverage.

3.2.3. Hydrogen peroxide scavenging activity.

The antioxidant potential of the polyherbal formulation was estimated to be $94.987 \pm 0.946 \%$ at $400 \mu \mathrm{g} / \mathrm{ml}$, and for ascorbic acid, it was obtained as $97.24 \pm 0.79 \%$ at 400 $\mu \mathrm{g} / \mathrm{ml}$. IC50 of the polyherbal formulation was found to be $102.967 \mu \mathrm{g} / \mathrm{ml}$, whereas IC50 of ascorbic acid was found to be $57.443 \mu \mathrm{g} / \mathrm{ml}$ (Figure 6).

3.2.4 Effect on phytochemical and physicochemical analysis.

The results of the physicochemical analysis showed the specimen used for the study have Glycosides, Flavonoids, Saponins, etc. (Table 1). The presence of these phytoconstituents indicates its antioxidant potential. 


\subsection{Discussion.}

In our present study, we explored antiurolithiatic activity of Dolichos biflorus seedsbased functional beverage using in vitro crystallization model of human urine. The crystallization of calcium oxalate was significantly reduced with our study formulation (functional beverage) of Dolichos biflorus seeds. Dolichos biflorus seeds are a rich source of various natural bioactive substances and have the potential for treating urolithiasis, including a variety of other metabolic diseases [15]. Although a definite mechanism of Dolichos biflorus seeds is yet to be explored, we planned this study and used Dolichos biflorus seeds as a functional beverage to explore the role of bioactive substances Dolichos biflorus seeds on the crystallization phenomenon of calcium oxalate urolithiasis.

Numerous researches have confirmed the antioxidant capabilities of various flavonoids, glycosides, and saponins found in plants. The presence of these phytoconstituents suggests that they may be employed as potential antioxidant agents. DPPH, Hydrogen peroxide, and Reducing power activity results indicate the significant antioxidant potential of Dolichos biflorus seeds-based functional beverage comparable to standard ascorbic acid, could be advantageous in treating renal diseases mediated due to oxidative stress [4]. Higher concentrations of Dolichos biflorus seeds-based functional beverage in these assays have shown free radicals scavenging activity. Reducing power activity of Dolichos biflorus seedsbased functional beverage, has shown its reduction potential to reduce potassium ferricyanide $\left(\mathrm{Fe}^{3+}\right)$ to potassium ferrocyanide $\left(\mathrm{Fe}^{2+}\right)$, which indicates its role as a strong antioxidant. Attenuating oxidative stress may be viewed as a significant strategy for slowing urolithiasis progression. A series of steps along with a favourable environment is necessary for the development of urolithiasis. The supersaturation of urine, with calcium and oxalate, is a prerequisite for crystallization to occur but not sufficient to produce calcium oxalate crystals $[21,22]$. Afterward, nucleation, growth, and crystal aggregation occur due to a to favorable environment, followed by adhesion and internalization of the crystal into the tubular epithelial cells [27, 28].

Many authors reported the in vitro effect of Dolichos biflorus seeds on the crystallization of calcium phosphate [16-20]. However, the effect of Dolichos biflorus in the human urine calcium oxalate crystal model is yet to be explored.

Our results showed that this formulation possesses antioxidant properties and inhibited crystal growth, as crystals size in study formulation samples was observed smaller than that of the particles found in control samples. This fact is also supported by a decrease in absorbance in study formulation samples than in control samples. The high turbidity is associated with increased/larger crystals and increased absorbance during the turbidimetric assay. Also, the samples containing study formulation have shown reduced crystal aggregation. The reduction in crystals aggregation helps prevent urolithiasis by keeping the crystals dispersed in the urine, controlling their size, and facilitating expulsion from the urinary tract.

In summary, we showed that Dolichos biflorus inhibited the calcium oxalate crystallization process by reducing crystal growth and aggregation.

\section{Conclusions}

Our study showed that Dolichos biflorus based functional beverage possess antioxidant properties and inhibited calcium oxalate crystallization process by reducing crystal growth and aggregation. Thus, the functional beverage of Dolichos biflorus seeds being food may be 
devoid of adverse effects and can be considered a potential candidate for treating calcium oxalate kidney stones.

\section{Funding}

This research received no external funding.

\section{Acknowledgments}

The authors are grateful to the Chitkara College of Pharmacy, Chitkara University, Rajpura, Patiala, Punjab, India, for providing the necessary facilities to carry out the research work.

\section{Conflicts of Interest}

The authors declare no conflict of interest.

\section{Ethics Approval}

All the experimental procedures and protocols used in this study were approved by the Institutional Animal Ethics Committee (ISFCP/IEC/2019/41).

\section{References}

1. Ormanji, M.S.; Rodrigues, F.G.; Heilberg, I.P. Dietary Recommendations for Bariatric Patients to Prevent Kidney Stone Formation. Nutrients 2020, 12, https://doi.org/10.3390/nu12051442.

2. Ahmad, W.; Khan, M.A.; Ashraf, K.; Ahmad, A.; Daud Ali, M.; Ansari, M.N.; Kamal, Y.T.; Wahab, S.; Zaidi, S.M.A.; Mujeeb, M.; Ahmad, S. Pharmacological Evaluation of Safoof-e-Pathar Phori- A Polyherbal Unani Formulation for Urolithiasis. Frontiers in Pharmacology 2021, 12, https://doi.org/10.3389/fphar.2021.597990.

3. Kant, R.; Singh, T.G.; Singh, S. Mechanistic approach to herbal formulations used for urolithiasis treatment. Obesity Medicine 2020, 19, https://doi.org/10.1016/j.obmed.2020.100266.

4. Saleem, U.; Ahmad, N.; Shah, M.A.; Anwar, F.; Ahmad, B. Anti-urolithiatic activity of Salvia hispanica L. seeds in ethylene glycol induced urolithiasis rat's model. An Acad Bras Cienc 2020, 92, https://doi.org/10.1590/0001-3765202020200067.

5. Youn, S.H.; Kwon, J.H.; Yin, J.; Tam, L.T.; Ahn, H.S.; Myung, S.C.; Lee, M.W. Anti-Inflammatory and Anti-Urolithiasis Effects of Polyphenolic Compounds from Quercus gilva Blume. Molecules 2017, 22, https://doi.org/10.3390/molecules22071121.

6. Assimos Dean, G. Urolithiasis/Endourology. Journal of Urology 2021, 205, 623-626, https://doi.org/10.1097/JU.0000000000001485.

7. Abu-Ghanem, Y.; Kleinmann, N.; Erlich, T.; Winkler, H.Z.; Zilberman, D.E. The Impact of Dietary Modifications and Medical Management on 24-Hour Urinary Metabolic Profiles and the Status of Renal Stone Disease in Recurrent Stone Formers. The Israel Medical Association journal 2021, 23, 12-16

8. Itoh, Y.; Yasui, T.; Okada, A.; Tozawa, K.; Hayashi, Y.; Kohri, K. Preventive effects of green tea on renal stone formation and the role of oxidative stress in nephrolithiasis. The Journal of urology 2005, 173, 271275.

9. Singh, T.G.; Singh, H.P.; Kaur, S.; Dhiman, S. Protective effects of sesamol against cisplatin-induced nephrotoxicity in rats: A mechanistic approach. Obesity Medicine 2020, 19, https://doi.org/10.1016/j.obmed.2020.100269.

10. Monti, E.; Trinchieri, A.; Magri, V.; Cleves, A.; Perletti, G. Herbal medicines for urinary stone treatment. A systematic review. Archivio Italiano di Urologia e Andrologia 2016, 88, 38-46, https://doi.org/10.4081/aiua.2016.1.38.

11. Topolska, K.; Florkiewicz, A.; Filipiak-Florkiewicz, A. Functional Food-Consumer Motivations and Expectations. International Journal of Environmental Research and Public Health 2021, 18, https://doi.org/10.3390/ijerph18105327.

12. Adefegha, S.A. Functional Foods and Nutraceuticals as Dietary Intervention in Chronic Diseases; Novel Perspectives for Health Promotion and Disease Prevention. Journal of Dietary Supplements 2018, 15, 9771009, https://doi.org/10.1080/19390211.2017.1401573. 
13. Dhaliwal, S.K.; Talukdar, A.; Gautam, A.; Sharma, P.; Sharma, V.; Kaushik, P. Developments and Prospects in Imperative Underexploited Vegetable Legumes Breeding: A Review. International Journal of Molecular Sciences 2020, 21, https://doi.org/10.3390/ijms21249615.

14. Amal, T.C.; Karthika, P.; Dhandapani, G.; Selvakumar, S.; Vasanth, K. A simple and efficient Agrobacterium-mediated in planta transformation protocol for horse gram (Macrotyloma uniflorum Lam. Verdc.). Journal of Genetic Engineering and Biotechnology 2020, 18, https://doi.org/10.1186/s43141-02000023-z.

15. Papp-Wallace, K.M.; Shapiro, A.B.; Becka, S.A.; Zeiser, E.T.; LiPuma, J.J.; Lane, D.J.; Panchal, R.G.; Mueller, J.P.; O'Donnell, J.P.; Miller, A.A. In Vitro Antibacterial Activity and In Vivo Efficacy of SulbactamDurlobactam against Pathogenic Burkholderia Species. Antimicrob Agents Chemother 2021, 65, e01930-20, https://doi.org/10.1128/AAC.01930-20.

16. Nirumand, M.C.; Hajialyani, M.; Rahimi, R.; Farzaei, M.H.; Zingue, S.; Nabavi, S.M.; Bishayee, A. Dietary Plants for the Prevention and Management of Kidney Stones: Preclinical and Clinical Evidence and Molecular Mechanisms. International Journal of Molecular Sciences 2018, 19, https://doi.org/10.3390/ijms19030765.

17. Bijarnia, R.K.; Kaur, T.; Singla, S.K.; Tandon, C. A novel calcium oxalate crystal growth inhibitory protein from the seeds of Dolichos biflorus (L.). The protein journal 2009, 28, 161-168, https://doi.org/10.1007/s10930-009-9179-y.

18. Saha, S.; Verma, R.J. Antinephrolithiatic and antioxidative efficacy of Dolichos biflorus seeds in a lithiasic rat model. Pharmaceutical Biology 2015, 53, 16-30, https://doi.org/10.3109/13880209.2014.909501.

19. Atodariya, U.; Barad, R.; Upadhyay, S,; Upadhyay, U. Anti-urolithiatic activity of Dolichos biflorus seeds. J Pharmacogn Phytochem 2013, 2.

20. Patel, V.; Acharya, N. Anti-urolithiatic activities of Macrotyloma uniflorum Mediated through Multiple Pathway. Indian Journal of Pharmaceutical Education and Research 2020, 54, 403-415, https://doi.org/10.5530/ijper.54.2.46.

21. Kane, T.D. Ureteroscopy for Urinary Calculi with or Without Ureteral Stents. AJN The American Journal of Nursing 2020, 120, https://doi.org/10.1097/01.NAJ.0000662840.58258.75.

22. Ormanji, M.S.; Rodrigues, F.G.; Heilberg, I.P. Dietary Recommendations for Bariatric Patients to Prevent Kidney Stone Formation. Nutrients 2020, 12, https://doi.org/10.3390/nu12051442.

23. Tang, G.Y.; Meng, X.; Gan, R.Y.; Zhao, C.N.; Liu, Q.; Feng, Y.B.; Li, S.; Wei, X.L.; Atanasov, A.G.; Corke, H.; Li, H.B. Health Functions and Related Molecular Mechanisms of Tea Components: An Update Review. Int J Mol Sci 2019, 20, https://doi.org/10.3390/ijms20246196.

24. Shang, A.; Li, J.; Zhou, D.-D.; Gan, R.-Y.; Li, H.-B. Molecular mechanisms underlying health benefits of tea compounds. Free Radical Biology and Medicine 2021, 172, 181-200, https://doi.org/10.1016/j.freeradbiomed.2021.06.006.

25. More-Krong, P.; Tubsaeng, P.; Madared, N.; Srisa-Art, M.; Insin, N.; Leeladee, P.; Boonla, C. Clinical validation of urinary indole-reacted calcium oxalate crystallization index (iCOCI) test for diagnosing calcium oxalate urolithiasis. Scientific Reports 2020, 10, https://doi.org/10.1038/s41598-020-65244-1.

26. Uddandrao, V.V.S.; Parim, B.; Saravanan, G. Evaluation of the Antioxidant and Antidiabetic Potential of the Poly Herbal Formulation: Identification of Bioactive Factors. Cardiovascular \& Hematological Agents in Medicinal Chemistry 2020, 18, 111-123, https://doi.org/10.2174/1871525718666200207103238.

27. Singh, A.-K.; Yadav, D.; Sharma, N.; Jin, J.-O. Dipeptidyl Peptidase (DPP)-IV Inhibitors with Antioxidant Potential Isolated from Natural Sources: A Novel Approach for the Management of Diabetes. Pharmaceuticals 2021, 14, https://doi.org/10.3390/ph14060586.

28. Torzewska, A.; Wiewiura, P.; Brodecka, D.; Szczerbiec, D.; Różalski, A. Potentially Probiotic Lactobacillus Strains Derived from Food Intensify Crystallization Caused by Proteus mirabilis in Urine. Probiotics and Antimicrobial Proteins 2021, 13, 441-452, https://doi.org/10.1007/s12602-020-09689-w. 